\title{
DISCRETE-EVENT SIMULATION AND AUTOMATIC DATA COLLECTION IMPROVE PERFORMANCE IN A MANUFACTURING SYSTEM
}

\author{
Arne Ingemansson \\ Jan Oscarsson \\ University of Skövde \\ Centre for Intelligent Automation \\ P.O. Box 408 \\ SE-541 28 Skövde \\ SWEDEN
}

\begin{abstract}
Data utilization from an automatic collection system helps simulation. DES (Discrete-Event Simulation) is a suitable tool in that respect. Improvements of a manufacturing system by reducing production disturbances in block engine line of a truck manufacturer were shown in a case study. The manufacturing line is equipped with an automatic data gathering system, collecting detailed information from each machine, such as DT (DownTime). The collected data and the simulation model were used in finding bottlenecks in the system. The improvement groups of the company suggested improvements to these machines in collaboration with DES work. An improvement of $12 \%$ was achieved in the block line during two years. The long-term improvement work was profitable. Further potential with the method is investigated in a new case study.
\end{abstract}

\section{INTRODUCTION}

A systematic approach to production disturbance reduction and thus improvement of efficiency in a manufacturing system is necessary to a company in today's competitive world. Production disturbances in manufacturing lines are a common industrial problem (Smet, Gelders, and Pintelon 1997). Previous research shows that of total production time only $50-60 \%$ is used for manufacturing and the rest of the time is wasted in different production disturbances (Ericsson 1997, Drucker 1990). In spite of the development of different tools and management techniques, the figure has not changed much. The increased complexity and variants of products in manufacturing lines may be one explanation.

Measurement of a manufacturing system is necessary in order to improve performance. A key problem in many projects is inadequate data. The case study has shown the potential of using automatic data collection from the dif- ferent machines in the manufacturing system in the DES model. Each machine in the line is equipped with equipment that can collect data direct from the PLC (Programmable Logic Controller) system of each machine in a manufacturing system. Among other things the DT (DownTime) data can be investigated in an accurate way. Previous difficulties of collecting accurate DT data have been shown (Williams 1994).

The findings in this paper indicate a potential in combining automatic collected data and DES work. A case study has showed an improvement of $12 \%$ in a two-year period. Downtime and to some extent cycle times were reduced by five different bottleneck studies. The system with automatic data collection has been installed on more manufacturing systems and gives reasons to work on a new case where the method is further generalized.

\section{MATERIAL \& METHODS}

\subsection{Increased Performance in Manufacturing}

Continuous improvements of manufacturing systems are necessary for a competitive industry. There are different ways to achieve increased performance. Manufacturing systems based on learning organizations will show possibilities to increase productivity (Bessant and Francis 1999). The manufacturing system must also be stable to produce efficiently. The most competitive production methods such as TPS (Toyota Production System) and their best components have been shown to be successful.

Follow-up of production disturbances, preventive maintenance and other improvement work are cornerstones of TPM (Total Productive Maintenance). DES is a useful tool in the improvement work. Stability is achieved by a process with as little production disturbances as possible. Case studies have shown the potential to increase efficiency in a manufacturing system by studying production disturbances (Ingemansson, 2004). 


\subsection{Overall Equipment Effectiveness, OEE}

A manufacturing system can be measured, among other things, in production, quality, time, cost and flexibility. The design of manufacturing system has often been based on empirical experience. The demand of increased capacity in manufacturing systems will change this to a more systematic method. OEE (Overall Equipment Effectiveness) is a suitable measuring method for semi or fully automated systems. Losses are classified as downtime, cycle and quality losses. Downtime losses can be divided into planned and unplanned stops. The different ideas of time allocation for equipment according to the OEE formula are shown in Figure 1.

Reduction of production disturbances helps increase efficiency in the manufacturing system. Downtime losses are a part of the system availability rate. Without doubt the easiest way to improve performance in a system is to reduce downtime losses and to increase the system availability rate. Surprisingly, the figure of OEE has not improved over the years according to the authors' experience. The question to be answered is why an overall improvement has not taken place in spite of all the new powerful tools and management techniques. One explanation may be the complexity; for example, the numbers of variants has often increased in the same manufacturing line.

\subsection{Measurement of Key Figures}

Measurement of data and key figures can give clues in the improvement work. Consistent measurement and also key figures are necessary to give an idea of how the manufacturing system is behaving. The basic data could be collected from automatic systems or manual measurements. It is important to measure as correctly as possible. However, it is more important to measure something and to be able to compare the figures to next week than not measure anything at all. The figures will give an indication, but the best is to measure as correctly as possible, although there is always a possibility to change a formula in a later stage. Different production disturbance causes can be identified and thereafter reduced. New ones have then to be identified to continue the reduction of disturbances. To measure production disturbances adequately is a key to the improvement work.

There are often rough data in the MRP (Manufacture Resource Planning) system. It can be used for validation but it is far too imprecise to be adequate for input data. An established method is to measure according to the OEE, see Figure 1. There is a trend to shorten the measurement intervals too; some companies even measure key figures on single shifts.

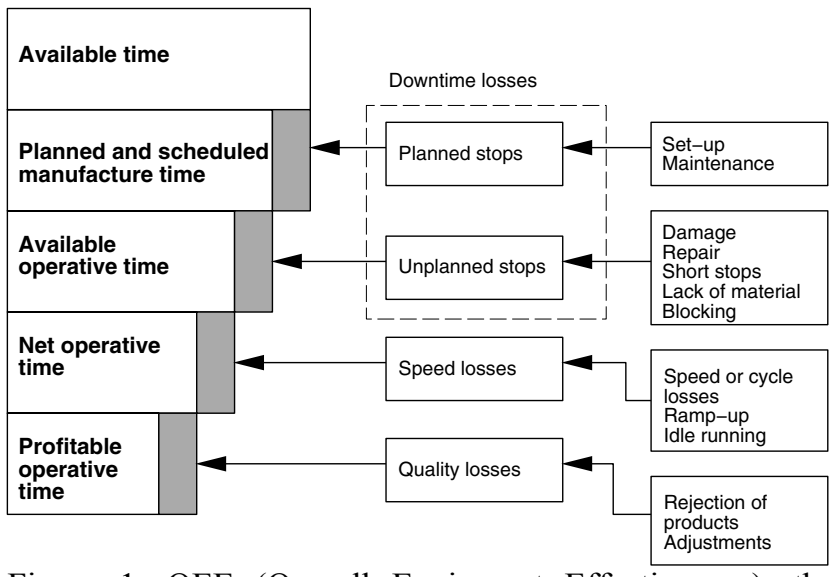

Figure 1: OEE (Overall Equipment Effectiveness), the Profitable Operative Time Is Often Small Compared To the Planned and Scheduled Manufacture Time; Unplanned Stops Decreases the Available Operative Time

\subsection{Discrete-Event Simulation}

The automatic data collection system gives all numeral data to the model. Still, all logics of the system have to be collected and included before there is a useable model. Many of today's manufacturing systems are designed bit by bit and it may sometimes be hard to discern the logics. On-site measurements are in most cases necessary for an adequate DES model. An advantage of this method is that it also gives valuable information from machine operators and other involved personnel.

The DES model has different fields of applications in improving capacity of a manufacturing system, for example: Fast playing of historic data to get an overview of the actions in the manufacturing system. Second, modifications in the manufacturing systems based on actual data, for example an elimination of a specific production disturbance, can also be a field of application. The model is shown in Figure 2 with the status highlighting turned on. The different colors of the machines indicate the status; green for busy, yellow for idle, red for failure and orange for blocked.

\subsection{Improvement Work}

Improvement work at a company must be a goal for an organization working with increased performance in manufacturing systems. Established routines must be standardized within the organization. Thus, time and resources must be allocated for this purpose. Improvement work is teamwork and some companies have for example weekly meetings for this purpose. Data and other relevant material have to be prepared before the meeting. The improvement groups must also keep records of their meetings. It is necessary to keep a log, for example a follow-up of changes in the manufacturing system. 


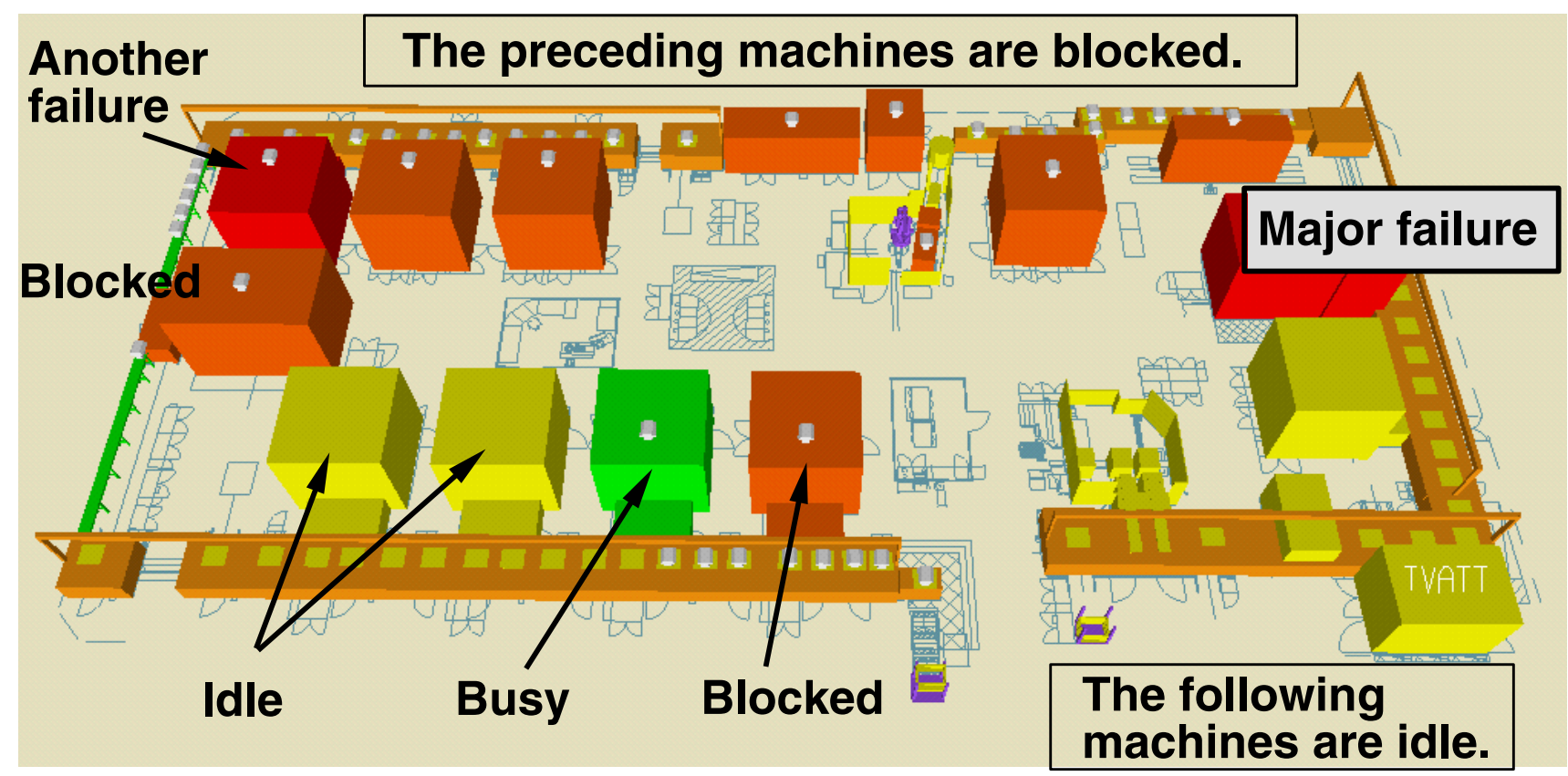

Figure 2. Machines Preceding a Failure Are Blocked and the Following Machines Are Idle

\section{THE CASE STUDY}

\subsection{Background}

A multinational company in Sweden manufacturing engine blocks has equipped the manufacturing system with an automatic data collection system. Different sets of data were logged automatically at each machine such as DT (DownTime) and TBDT (Time Between DownTime). The conditions of all machines are read more than once per second. Reasons for each DT were also included automatically from each machine insofar as possible in the manufacturing line. The manufacturing system includes $11 \mathrm{NC}$ (Numerically Controlled) machines and six other stations including assembling, washing and cleaning, and quality control. The NC-machines mill and drill the blocks. At the output the block is ready for the motor assembly line.

The company has implemented "lean production". Buffers should be reduced as far as possible. In the manufacturing line there is only one area with buffers. It is a delicate problem to decide the suitable number of buffers in a manufacturing system. Too many buffers will lead to a high cost if something in the manufacturing system goes wrong and the manufactured parts have to be altered or discarded. The real problems may be hidden and there are also the questions about how much capital should be tied up. TPS tries to minimize the buffers in order to find problems as quickly as possible.

\subsection{The Improvement Work}

A bottleneck analysis is often an appropriate method for improving performance in a DES model. The bottleneck was identified by studying the simulation runs and was verified by the statistics from the model. Improvements of this single $\mathrm{NC}$ machine were shown to improve the whole system. A general method to find a bottleneck in a manufacturing system may be to find a machine with many production disturbances and a high level of utilization. The consequence of a longer production disturbance is shown in Figure 2. The simulation model can show the sequence both backwards and forwards in the manufacturing chain. Upstream from the stopped machine the machines are blocked and downstream the machines are idle. Longer production disturbances must be avoided. The example shown is an extreme case to illustrate the consequence and is not considered to be normal production at the company.

\section{RESULTS}

The results from the case study showed the improvement work with DES was beneficial, see Table 1 . A capacity increase of $12 \%$ has been seen in a two-year period. The increase has continued after this period. No major investments were made; the increase in capacity has been made by different working methods and tools. Engineering time has been used in analysis, simulation, and implementation of improvements. 
Table 1: Increase in Overall Output of the Studied Manufacturing System

\begin{tabular}{cc}
\hline Project time & $\begin{array}{c}\text { Capacity improvement, } \\
\text { output increase }\end{array}$ \\
\hline 12 months & $6 \%$ \\
24 months & $12 \%$ \\
\hline
\end{tabular}

The bottleneck machine was also studied manually on site together with the DES work. The manual data gave valuable information to improve the machine. Examples of improvements in the machines were implementation of new tools of longer useful life and consequently longer replacement intervals.

\section{DISCUSSION}

To reduce or eliminate production disturbances is one of the best ways to increase efficiency in the system. In any case, limited categories of production disturbances should be used and logged. Ten different production disturbances are suggested by the authors of this paper and the different classes should be well distinguished from each other. Furthermore, the unclassified events must be lower than $10 \%$. The consistency related to production disturbances at a manufacturing line, for example classification and followup, are previously emphasized (Harlin, Ylipää \& Fjällström 2002).

Follow-up of production disturbances combined with DES may enable a more efficient manufacturing system. Better measurement of the manufacturing systems is a condition for increased performance. Automatic data collection and the experience of the machine operators help improve the quality of production disturbance data. Time was adequately logged in the case study and there is an ongoing project of logging the different background causes of production disturbances, too.

If the reasons for the production disturbances are logged, much better background data will be provided. One idea is to use adequate pre-selected reasons of production disturbances for the machine operators to choose from. Then different background causes of production disturbances can be logged for easy follow-up. Other reports (Ingemansson 2004) indicate further the potential of the method.

Some issues with the automatic data collection system have yet to be solved. There are today unexplained short stops logged. Therefore, all status logged are not valid in the database. Other questions are about a fully automated transfer of data between the database and the DES model to, for example, model a week's production to find the current bottleneck in the system. The person suitable for this work, for example the production engineer at each department and his role at the company has also to be decided.

\section{CONCLUSIONS}

To combine automatically collected data from the different machines in a manufacturing system and use the information in a DES model is a profitable solution. Bottleneck analyses together with manual studies on site gave ideas for improvements, and with low investments. The case study showed a $12 \%$ increase in capacity in a two-year period. The automatic data can also be supplemented by the background reasons for the production disturbances which will be the case in a future case study.

\section{FUTURE WORK}

A new project has been initiated at the company. Another manufacturing system is studied with a semi-automatic data collection system. One of the goals of the project is to generalize the use of the results for further implementation with same method in different manufacturing lines throughout the company. Some technical questions about the automatic data collection system must also be solved. In the future, DES may be a more common improvement tool used together with the automatic data collection system.

\section{ACKNOWLEDGMENTS}

The authors have participated in the Swedish research project VINNOVA, Swedish Agency for Innovation Systems. We are also grateful to the company "B4Industry" for the help with the data acquisition.

\section{REFERENCES}

Bessant, J., and Francis, D. 1999. Developing strategic continuous improvement capability. International Journal of Production Management 19 (11) 11061119.

Drucker, P. F. 1990. The emerging theory of manufacturing. Harvard Business Review. May-June.

Ericsson, J. 1997. Störningsanalys av tillverkningssystem: Ett viktigt verktyg inom Lean Production. PhD Thesis, Lund University, Department of Production and Materials Engineering. In Swedish.

Harlin, U., Ylipää, T., and Fjällström S. 2002. Production Disturbance Handling from an Operative Perspective in Advanced Manufacturing Systems. Nordic Ergonomics Society 34th Annual Congress.

Ingemansson, A. 2004. On reduction of production disturbances in manufacturing systems based on discreteevent simulation. $\mathrm{PhD}$ Thesis, Lund University, Department of Mechanical Engineering.

Smet, R. D., Gelders, L. and Pintelon, L. 1997. Case studies on disturbance registration for continuous improvement. Journal of Quality in Maintenance Engineering 3 (2), 91-108. 
Williams, E. J. 1994. Downtime data - its collection, analysis, and importance. Proceedings of the Winter Simulation Conference, eds. J.D. Tew, S. Manivannan, D.A. Sadowski, and A.F. Seila, 1040-1043.

\section{AUTHOR BIOGRAPHIES}

ARNE INGEMANSSON is working in a research project at the Centre for Intelligent Automation at the University of Skövde, Sweden. He received his Ph.D. in 2004 at Lund University, Sweden. His research interests include improvements of manufacturing systems combined with production improvement methods and discrete-event simulation based on different case studies. His e-mail address is arne.ingemansson@his.se.

JAN OSCARSSON is a senior lecturer at the Centre for Intelligent Automation at the University of Skövde, Sweden. He received his Ph.D. 2000 at De Montfort University, Leicester, UK. His main research interests includes integrated product development, the 'digital factory' concept and issues related to systems engineering. His email is jan.oscarssonahis.se and Web addresses is http://www.his.se/templates/vanligwebbs ida1.aspx?id=18159. 